\title{
How to Process Zillions of Spectra from Spectral Imaging Datasets? From Phase-Mapping to Bulk-Chemistry on Micron- to Centimeter Scale Using PARC.
}

\author{
Corrie van Hoek
}

Tata Steel Europe, R\&D, Ceramics Research Centre (CRC), P.O. Box 10.000, 1970 CA IJmuiden, The Netherlands

Scanning Electron Microscopy (SEM) combined with microanalysis is widely used for characterization of solid materials. With the current generation of hardware, 1) the intense Field Emission Gun beams, 2) Energy Dispersive X-ray analysis system using Silicon Drift Detectors (SDD) and 3) the fast acquisition hardware technology, the chemical information from each pixel in the SEM field can be acquired within microseconds. In other words, for a typical field of 512x384 pixels a Spectral Image (SI) of sufficient quality for quantitative analysis can be obtained in five minutes. The greatest challenge now is how most efficiently to extract maximum information out of all these zillions of spectra. At CRC we have developed the post-processing software PARC for precisely this purpose.

The standard SI data processing software does not fully harness the wealth of information stored in SI data sets. The established approach is typically as follows: 1) element distribution maps are produced with the option of multiple element overlays, 2) limited phases are assigned based on individual elemental thresholds or 3) principle "spectral" components are derived using multi-variant statistics. Such approaches have severe drawbacks. Clearly ZAF correction is not applicable to element maps or principle components - hence the failure of these methods to quantify the phase constituents of materials. In addition the options for batch data evaluation are limited. In contrast, the aim and achievement of PARC is PhAse Recognition and Characterization, as captured in the acronym. PARC assigns individual pixel spectra to groups corresponding with true phases, allowing ordinary ZAF corrections to be confidently applied to the summed spectrum for each pixel grouping. Batch processing of SI datasets is also integral to its functionality. The following consistency checks are available for validation of results: 1) the quantified chemistry of PARC-defined crystalline phases should correspond to known stoichiometry, 2) the inventory of PARC-defined phases and their proportions should be reproducible with Powder X-ray diffraction - Rietveld analysis, 3) PARC results (chemical compositions and area proportions of phases), in combination with appropriate (assumed) phase densities, allow the bulk chemical composition of the analyzed area to be calculated and compared with independent bulk chemical analyses. These three validation methods are routinely applied, as will be illustrated during the presentation.

Two strategies can be followed in PARC to process SI data sets, the full-automatic mode and the query mode. In full-automatic mode PARC sorts the individual pixel spectra according to their characteristic multi-element combinations using only two constraints: peak threshold above background and a lower $\mathrm{X}$-ray energy limit cut-off. Generally this is a successful strategy with coarse materials having phase domains of approx. $20 \mu \mathrm{m}$ or larger, such as e.g., geological samples [1] and metallurgical slags. In full-automatic mode, phases forming solid solutions with a broad chemical range can become separated in the phase assignment but can be manually merged afterwards. Compositional gradients can be highlighted in solid solution phases for a single element, ratio or combination of elements of interest. The full-automatic mode faithfully recognizes trace phases with unusual elements, or combinations of elements. Artifacts associated with adjoining phase boundaries can be easily subdued applying image 
processing techniques such as erosion operations on peripheries of phases. In the other strategy, the query mode, PARC is asked to search for known element combinations that are specific for a given phase of interest. This works well with prior knowledge of a sample, e.g. from XRD-analysis. Typically, one phase after another is assigned and remaining white (unidentified) pixels in the PARC Phase image are subsequently checked for their element combination, leading to new queries for sorting and phase assigning to be applied on the remaining unidentified pixels.

Once a PARC model has been established it can be run in batch on series of SI fields. This is where great time saving in data processing can be achieved. To illustrate, in a particularly challenging problem associated with ore-pretreatment for the Blast Furnace, the examination of cross-sections of $15 \mathrm{~mm}$ diameter iron-ore pellets was required to quantify reaction progress during a high temperature induration process. This required SI data acquisition and processing for 160 different pellet cross sections consisting of 120 SI fields each time, with each field comprising $512 \times 384$ pixels at a resolution of ca. $0.5 \mu \eta$ per pixel. The total SI data acquisition time was $1600 \mathrm{hrs}$, processing the ca. $3.8 \times 10^{9}$ individual pixel spectra with PARC took $640 \mathrm{hrs}$ and the whole job was completed in 14 weeks of which the majority of the acquisition and processing tasks were done overnight and weekends.

Another recurring problem for heavy industry is fugitive dust causing complaints in the environs of a production plant. Dust poses a particular challenge because both particle diameter and phase composition are of interest. Airborne dust transport is restricted to small particles generally below 100 micron. Each individual particle is normally too small to yield a representative composition of the source material, because it is usually only fragments of one or more phases of the source material. Using PARC for phases mapping on 3D particles, populations of particles with phase combinations related to a single source can be recognized, as well as multiple different populations related to different sources. With this type of approach the various sources contributing to dust fallout can be analyzed [2]. An example of dust characterization is presented in Figure 1.

[1] C van Hoek et al, Microscopy and Microanalysis 17-5 (2011) p. 656.

[2] C van Hoek et al, European Microbeam Analysis Society (2013) p. 405

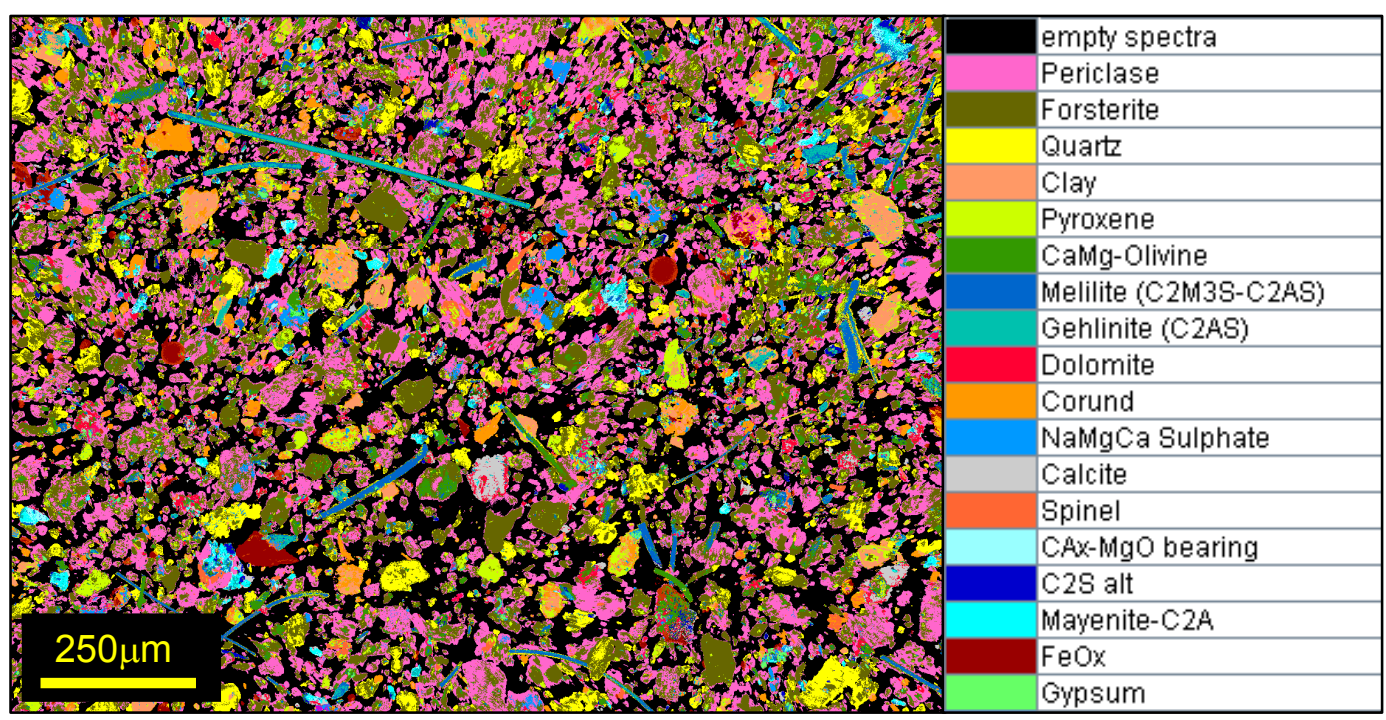

Figure 1. SI-Phase Mapping of dust particles 